\title{
Insulin therapy and lipoproteins in patients with type 1 diabetes
}

\begin{abstract}
Knowledge on HDL cholesterol has been extended, however, there are still unknown facts, especially concerning HDL cholesterol, type 1 diabetes mellitus and insulin therapy. It is known that HDL is responsible for the transport of cholesterol to the liver. It is called the "good cholesterol" due to its advantageous properties. HDL cholesterol reduces cardiovascular risk by its anti-inflammatory, antioxidant, profibrinolytic and antithrombotic effects. The influence of HDL on the incidence of chronic complications of type 1 diabetes has not been yet fully understood. The researchers observed an increase in HDL cholesterol level after initiation of insulin therapy. Significant is the fact that insulin affects lipoprotein metabolism in type 1 diabetes, as it is the treatment of choice in this group of patients. (Clin Diabetol 2016; 5, 4: 111-116)
\end{abstract}

Key words: lipoproteins, type 1 diabetes, HDL cholesterol, insulin therapy, chronic diabetic complications

\section{Introduction}

Type 1 diabetes (DM1) is an autoimmune disease. Nowadays, the incidence of DM1 is still increasing. Due to absolute insulin deficiency of patients with DM1, insulin therapy is the treatment of choice.

Address for correspondence: lek. Aleksandra Cieluch

Katedra i Klinika Chorób Wewnętrznych i Diabetologii

Uniwersytet Medyczny w Poznaniu

Szpital Miejski im. Franciszka Raszei

ul. Mickiewicza 2, 60-834 Poznań

Phone: 661295634

e-mail: aleksandra.cieluch@o2.pl

Clinical Diabetology 2016, 5, 4, 111-116

DOI: $10.5603 /$ DK.2016.0019

Received: 31.05 .2016
Intensive functional insulin therapy (IFI) is a recommended method of treatment for patients with DM1. It mimics physiological pattern of insulin secretion. Moreover, it has been shown to be superior to conventional insulin therapy in terms of metabolic control of the disease. IFI involves adjusting doses of short-acting insulin depending on the blood glucose levels before meals, the amount of planned carbohydrate exchangers consumption and anticipated exercise [1].

The Diabetes Control and Complication Trial (DCCT) has shown the relationship between better glycaemic control in patients treated with intensive insulin therapy and lower risk of chronic diabetic complications such as peripheral neuropathy, retinopathy, nephropathy and angiopathy [2]. Moreover, this benefit effect persist long time of observation despite worsening of glycaemic control.

The good metabolic control of diabetes is a very important goal of treatment. It focuses not only on normoglycaemia, but also on lipid profile, blood pressure and body mass index (BMI) (Tab. 1).

\section{The lipoproteins}

Cholesterol, triglycerides (TG) and phospholipids are transported in the blood as lipoprotein complexes,

Table 1. Criteria for the lipid control in patients with diabetes (Polish Diabetes Association guidelines 2016) [3]

\begin{tabular}{lcc}
\hline & [mg/dL] & {$[\mathrm{mmol} / \mathrm{L}]$} \\
\hline Total cholesterol & $<175$ & $<4.5$ \\
LDL cholesterol & $<70$ & $<1.9$ \\
LDL cholesterol in young patients & $<100$ & $<2.6$ \\
with DM1 (<40 years) & & \\
HDL cholesterol* & $>40$ & $>1.0$ \\
"Non-HDL cholesterol" & $<130$ & $<3.4$ \\
Triglycerides & $<150$ & $<1.7$ \\
\hline
\end{tabular}

*For women increase by $10 \mathrm{mg} / \mathrm{dL}$ (about $0.275 \mathrm{mmol} / \mathrm{L}$ )

LDL — low density lipoproteins; HDL — high density lipoproteins 
Table 2. The characteristic of lipoproteins [4]

\begin{tabular}{|c|c|c|c|c|c|c|c|}
\hline \multirow[t]{2}{*}{ Lipoprotein } & \multirow[t]{2}{*}{ Source } & \multirow{2}{*}{$\begin{array}{c}\text { Diameter } \\
{[\mathrm{nm}]}\end{array}$} & \multirow{2}{*}{$\begin{array}{l}\text { Density } \\
{[\mathrm{g} / \mathrm{mL}]}\end{array}$} & \multicolumn{2}{|c|}{ Composition (\%) } & \multirow{2}{*}{$\begin{array}{l}\text { Major lipid } \\
\text { components }\end{array}$} & \multirow[t]{2}{*}{ Apolipoprotein } \\
\hline & & & & Protein & Lipid & & \\
\hline Chylomicrons & Intestine & $90-1000$ & $<0.95$ & $1-2$ & 98-99 & Triacylglycerol & A-I, A-II, AIV, B-48 \\
\hline $\begin{array}{l}\text { Chylomicron } \\
\text { remnants }\end{array}$ & Chylomicrons & $45-150$ & $<1.006$ & $6-8$ & $92-94$ & $\begin{array}{l}\text { Triacyloglycerol, phos- } \\
\text { pholipids, cholesterol }\end{array}$ & $B-48$ \\
\hline VLDL & $\begin{array}{l}\text { Liver and } \\
\text { intestine }\end{array}$ & $30-90$ & 0.95-1.006 & $7-10$ & $90-93$ & Triacyloglycerol & $\begin{array}{l}\text { B-100, C-I, } \\
\text { C-II, C-III }\end{array}$ \\
\hline IDL & VLDL & $25-35$ & $1.006-1.019$ & 11 & 89 & $\begin{array}{c}\text { Triacyloglycerol, } \\
\text { cholesterol }\end{array}$ & $B-100, E$ \\
\hline LDL & VLDL & $20-25$ & $1.019-1.063$ & 21 & 79 & Cholesterol & B-100 \\
\hline HDL1 & Liver, intestine, & $20-25$ & $1.019-1.063$ & 32 & 68 & Phospholipids, & A-I, A-II, AIV, C-I, \\
\hline HDL2 & VLDL, & $10-20$ & $1.063-1.125$ & 33 & 67 & cholesterol & C-II, \\
\hline HDL3 & chylomicrons & $5-10$ & $1.125-1.210$ & 57 & 43 & & C-III, D, E \\
\hline pre- $\beta$-HDL & & $<5$ & $>1.210$ & & & & $A-I$ \\
\hline Albumin/FFA & Adipose tissue & & $>1.281$ & 99 & 1 & FFA & \\
\hline
\end{tabular}

VLDL — lipoproteins of very low density; IDL — intermediate density lipoproteins; LDL — low density lipoproteins; HDL — high density lipoproteins; FFA - free fatty acids

while the free fatty acids (FFA) with albumins. According to size, we can distinguish 6 groups of lipoproteins: chylomicrons, the particles of residual chylomicrons, lipoproteins of very low density (VLDL), intermediate density lipoproteins (IDL), low density lipoproteins (LDL) and high density lipoproteins (HDL) (Tab. 2). Metabolism of lipoproteins can be divided into endogenous and exogenous pathways. The former are responsible for transporting lipids from the intestine to the liver, the latter transfer lipids between tissues.

In the metabolic pathway of exogenous nutrients, TG-rich chylomicrons are converted by lipoprotein lipase (LP) to residual chylomicrons particles containing cholesterol esters, which are transferred to the liver, where they are degraded in lysosomes. Lipoprotein lipase is an enzyme present on the surface of the capillary endothelium, removing chylomicrons from the circulation. It catalyzes the degradation of TG in chylomicrons to FFA and glycerol undergoing re-esterification after penetration into the fat cells.

In the endogenous pathway TG-rich VLDL are produced in the liver and they are transformed into IDL by LP. In further process, IDL are converted to rich in cholesterol esters LDL. The cholesterol esters are produced in the HDL by the action of lecithin-cholesterol acyltransferase (LCAT) and captured finally by the IDL. The LP also removes TG from the circulating VLDL.

LDL supplies lipids to the tissues, where they are oxidized and to the adipose tissue where they are stored. The fat is taken from the adipose tissue as FFA-related blood albumins. HDL transport cholesterol from the tissues to the liver. Cholesterol is finally excreted to the bile. This mechanism is responsible for lowering cholesterol concentration in the blood. The LDL modified by oxidation are also captured by the macrophages, this is important in the pathogenesis of atherosclerosis. The function of VLDL is to transfer TG produced from the fatty acids and carbohydrates in the liver to the extrahepatic tissues.

\section{Characteristics of HDL}

$\mathrm{HDL}$ is a lipoprotein containing $25 \%$ of total serum cholesterol in humans. The reference values are 1.2-2.0 $\mathrm{mmol} / \mathrm{l}$ in women and $1.1-1.8 \mathrm{mmol} / \mathrm{l}$ in men. Reduced levels of HDL are observed in patients with insulin resistance state like obesity and type 2 diabetes [5].

HDL particles are synthesized in the liver and the intestines. They provide a set of smaller molecules differing in parameters such as size, density and ratio of the lipid to protein composition or physico-chemical properties (Tab. 2). HDL particle is composed of lipids (45\%), with quantitative advantage cholesterol esters over triglycerides, as well as proteins, called apolipoproteins (55\%). The major protein components are: apo AI, A-II, A-IV, CI, C-II, C-III, D, E, J. Apo Al and apo A-II represent $90 \%$ of all proteins contained in the HDL molecule in ratio of $4: 1$. On the basis of used methodology several classifications of HDL lipoproteins can be established. Depending on the density of HDL particle, we can distinguish the following sub-classes:

- HDL 2 - large, containing 3-4 times more cholesterol esters and triglycerides;

- HDL 3; 
Table 3. Factors affecting the lipoprotein HDL. Adapted and modified from "The biology of HDL lipoprotein and its antisclerotic activity" [6]

\begin{tabular}{ll}
\hline Factors increasing level of HDL & Factors lowering level of HDL \\
\hline Low body weight & Obesity, low physical activity \\
Female & Male gender, cigarette smoking \\
Physical activity & High carbohydrate diet \\
Ethanol in small amounts & Diabetes, kidney disease, liver failure \\
Deficiency HMG-CoA reductase & Drugs: beta-blockers, thiazides, probucol \\
Estrogens, glucocorticosteroids & Androgens, progestogens \\
Fibrates and niacin & Isolated low HDL, gene mutation apo A-I, \\
High-fat diet & Tangier disease \\
\hline
\end{tabular}

HMG-CoA - 3-hydroxy-3-methylglutaryl-coenzyme A; HDL — high density lipoproteins

- lower HDL subclass $\rightarrow$ HDL2a, HDL2b, HDL3a, HDL3b, HDL3c;

- HDL 1;

- HDL 4.

Another classification is based on the physical and chemical characteristics of HDL particles and distinguishes 5 classes: very large, large, medium, small, very small HDL particles. Regarding protein content of the HDL molecule it can be divided into:

- HDL containing proteins of apo A-I and apo A-II;

- HDL containing only apo A-I.

There are many factors that influence the size of $\mathrm{HDL}$ particles, which may increase or decrease the size. Among these are: LCAT, phospholipid transfer protein (PLTP), and cholesterol ester transfer protein (CETP), hepatic lipase (HL) and phospholipase A2 (PLA2). Level of plasma HDL may also be modulated by various factors. Low body weight, physical activity, estrogen, insulin, high-fat diet increase its level. Obesity, high carbohydrate diet and diabetes (insulin resistance) reduce the level of HDL (Tab. 3).

It should be emphasized that HDL lipoproteins have beneficial effects on the human body. There is no doubt that the most important part of their action is the anti-atherosclerosis effect, and thus a beneficial effect on the cardiovascular system. This is achieved by involving HDL particles in the reverse transport of free cholesterol from peripheral cells to the liver. In addition, HDL inhibits the oxidative modification of LDL, exerts anti-inflammatory effects and stimulates prostaglandin synthesis, having together beneficial effects on reducing cardiovascular risk [6, 7].

Disorders of lipoprotein metabolism are present in many diseases. It appears that such disturbances are especially common in diabetes. A key development is the absolute or relative insulin deficiency (insulin resistance), which increases the excessive use of FFA and reduces consumption of chylomicrons and VLDL. This in turn, leads to elevated TG.
Insulin is a hormone regulating the mobilization of fats. It inhibits the release of FFA from adipose tissue, which reduces their concentration in plasma. Moreover, it stimulates lipogenesis and synthesis of acylglycerol. These phenomena are the result of insulin action on glucose uptake by adipocytes through GLUT 4 (glucose transporter type 4). The main activity of insulin in adipose tissue is the inhibition of hormone sensitive lipase, which reduces the release of FFA and glycerol. Therefore, insulin has an antilipolytic activity and highly increases HDL cholesterol levels.

\section{The effect of insulin therapy on lipid profile}

Insulin contributes to FFA synthesis in the liver. This phenomenon corresponds to the stimulating effect of insulin on glycogen synthesis. During this process, the liver is saturated with glycogen. The liver is no longer able to capture molecules of glucose and therefore directs them to the path, leading to the synthesis of fatty acids. The fatty acids are then transported to the liver as lipoproteins. In the circulation, lipoproteins are detached, what provides FFA to other tissues, including adipocytes, which consume FFA to the synthesis of TG.

Insulin inhibits lipolysis in adipose tissue, through the down regulation of the intracellular lipase. The lipase is responsible for the hydrolysis of the TG, and the release of fatty acids. Insulin assists in the displacement of glucose into the adipose tissue, where it is used to synthesize glycerol. On the other hand, glycerol and fatty acids are used to the synthesis of TG in adipocytes. This leads to further accumulation of TG in fat cells. Insulin stimulates lipogenesis and inhibits lipolysis.

Insulin influences the increase of HDL in patients with type 2 diabetes. Schmitt et al. have observed decreased levels of HDL cholesterol after treatment change from insulin to chlorpropamide. However, after 
the restoration of insulin therapy, the authors showed an increase in HDL cholesterol values [8].

Sinha et al. compared change in lipid parameters in patients with type 2 and DM1 after introduction of insulin therapy. It turned out that for patients with DM1, insulin therapy is beneficial to all lipids component. In patients with type 2 diabetes no such relationship was revealed [9].

In the DCCT study, lipid parameters among DM1 patients were analyzed. After 6.5 years of observation, the level of total cholesterol (TC) and the level of LDL cholesterol were slightly increased in patients treated with insulin, regardless of the method. Comparison of TC in both groups, intensive and conventional, revealed higher increase in patients treated with conventional method. The EDIC study compared lipid parameters in patients over the next 13-14 years. Long-term observation of the same patients showed different results than those presented in the DCCT. In particular, patients in both groups presented lower TC and LDL cholesterol, not only compared to the results after 6.5 years of the DCCT, but also compared to baseline [2].

Wilson et al. in the study of 16 young patients with DM1 observed changes in the concentration of plasma lipoproteins due to the intensification of insulin therapy. Patients were prescribed preprandial insulin doses, and taking extra doses of NPH (Neutral Protamine Hagedorn) insulin at bedtime. Before the intensification of treatment, patients were receiving only two doses of insulin per day, consisting of NPH insulin and short-acting insulin. The proper diet was applied. Thus, a measurement of lipid metabolism was made respectively after 3 and 6 months. A significant increase in HDL cholesterol after the intensification of insulin therapy has been noted, while the concentration of TC and TG did not change [10].

Guy et al. showed that in both, patients with well controlled DM1 $\left(\mathrm{HbA}_{1 \mathrm{c}}<7.5 \%\right)$ and patients with worse glycaemic control $\left(\mathrm{HbA}_{1 c} \geq 7.5 \%\right)$, HDL cholesterol is higher when compared to healthy controls. Patients with poor glycaemic control were treated with higher doses of insulin than patients with wellcontrolled blood glucose. Among the examined groups of patients the highest concentration of HDL cholesterol was observed among patients with higher $\mathrm{HbA}_{1 \mathrm{c}}$ [11]. That poses a question: is the increase of HDL cholesterol directly related to the dose of insulin?

Education is inherent in the treatment of patients with DM1. It should not only refer to the theoretical knowledge of the disease, but also to practical skills. Knowledge should be handed over in a progressive and systematic way, while concurrently controlling the state of the patients. It has been shown that patients, who scored better result in the diabetic knowledge test, achieve better treatment outcomes. Regarding the assessment of lipid parameters, better educated patients had higher HDL and lower TC and LDL cholesterol compared with patients who achieved a lower score in the test [12]. Araszkiewicz et al. showed that the use of IFI as well as having adequate knowledge of DM1, resulted in improved therapeutic effect.

It still has not been learned what are the mechanisms responsible for HDL cholesterol increase due to insulin therapy. It is also unclear which subtypes of HDL are affected with insulin and whether this increase in HDL cholesterol influences the development of chronic complications of DM1.

\section{Influence of lipids profle}

\section{on complications of type 1 diabetes}

Despite advances in the treatment of DM1, chronic diabetes complications remain a serious clinical problem. The development of complications in DM1 is influenced by many factors. These include genetic factors, duration of diabetes, hyperglycaemia, hypertension and smoking. It was also demonstrated that lipid abnormalities affect the occurrence of chronic complications in patients with DM1.

In patients with DM1 who meet all the diagnostic criteria for metabolic syndrome (MS) (the criteria by National Cholesterol Education Program Adult Treatment Panel III (NCEP-ATP III) microvascular complications were present in all of the patients. It is interesting that the criteria for MS diagnosis, in addition to glycaemic level, most frequently show low HDL cholesterol [13].

Rogowicz-Frontczak et al. demonstrated that dyslipidemia is a significant factor in the development of microvascular complications (retinopathy, nephropathy and neuropathy) in patients with DM1. Predictors of the occurrence of diabetic microangiopathy have been identified, showing that beyond the duration of diabetes, systolic and diastolic blood pressure, postprandial glucose, $\mathrm{HbA}_{1 \mathrm{c}}$ levels also elevated serum TG and LDL cholesterol levels were responsible for the increased risk of angiopathy [14].

During over a 7-year follow-up of patients with DM1 it has been observed that patients who developed autonomic neuropathy (CAN, cardiac autonomic neuropathy) were older, their body weight was greater, had longer history of diabetes, had a higher waist-to-hip ratio (WHR), systolic blood pressure, levels of $\mathrm{HbA}_{1 c^{\prime}} \mathrm{TC}$, LDL cholesterol, serum TG and lower HDL cholesterol levels when compared with patients without the CAN. Most patients suffered from albuminuria and retinopathy. However, after inspection and evaluation of risk factors of CAN it turned out that neither the duration of diabetes nor lipid parameters are no longer associated 
with the risk of $\mathrm{CAN}$. $\mathrm{HbA}_{1 \mathrm{c}}$ and hypertension were only predictors of CAN development [15].

Sun et al. have attempted to determine the incidence of complications in type 1 diabetic patients with disease duration over 50 years. Among the evaluated risk factors affecting the occurrence of angiopathy, the authors assessed the lipid profile. They noted correlation between reduced HDL cholesterol level and the increased incidence of cardiovascular disease. This drew attention to the relationship between elevated levels of sub pre- $\beta_{2}$ HDL with the presence of all diabetes complications, especially nephropathy and cardiovascular disease [16].

Soedamah-Muthu et al. investigated the relationship between the prevalence of risk factors and the occurrence of complications in patients with DM1. The authors took into account the age, $\mathrm{HbA}_{1 c^{\prime}}$ waist-to-hip ratio (WHR), the albumin/creatinine ratio, HDL cholesterol and other lipid parameters. It turned out that beyond the HDL cholesterol, all other factors were associated with the occurrence of diabetic complications. The survey results show that the higher HDL cholesterol, the lower risk of development of diabetic angiopathy. On the other hand, it was shown that the higher "non-HDL cholesterol" concentration, the greater diabetic complications risk [17].

Molitch et al. assessed the levels of lipoproteins in type 1 diabetic patients with micro- and macroalbuminuria, paying particular attention to HDL cholesterol. The authors have shown that patients with albuminuria had significantly lower HDL cholesterol levels compared to the patients without albuminuria. Patients with complications had worse level of glycaemic control. When analyzing the TC, it does not differ in both groups of patients. The concentration of LDL cholesterol and TG were higher among patients with albuminuria but did not reach statistical significance. According to the authors, the high concentration of HDL cholesterol can be a protective factor in the development of albuminuria in patients with DM1 [18].

Wierusz-Wysocka et al. obtained similar observations showing lower levels of HDL cholesterol in patients with diabetic retinopathy realted to DM1 compared with patients without this complication. High concentrations of HDL have been associated with 5-fold less risk of retinopathy. No difference was noted in the $\mathrm{HbA}_{1 \mathrm{c}}$ level between the two groups. This is another study that shows that high levels of HDL cholesterol may protect patients with DM1 from the development of diabetic microangiopathy [19].

Recent studies focus on HDL subclasses in relation to chronic diabetes complications. In a study of Lyons et al. [20] an inverse relation between HDL cholesterol level and retinopathy was found. In further analysis authors used nuclear magnetic resonance lipoprotein subclass profile and observed that the above mentioned relation was, in men, strong negative with the large HDL subclass and strong positive with the small HDL (considered potentially proatherogenic) subclass. In another study by Tolonen et al. [21] an inverse association between HDL and HDL2 and proliferative diabetic retinopathy was found. A study by Medina-Bravo et al. [22] provided an insight in HDL subclass distribution in relation to glycaemic control. Patients with good metabolic control were characterized by high levels of large (HDL2b and HDL2a) and low levels of small HDL (HDL3b and HDL3c) subpopulations. In multivariate regression analysis, in diabetic type 1 patients with poor metabolic control association with lower levels of large HDL2b was found. The above mentioned studies confirm positive association between diabetic complications, poor metabolic control and higher levels of small HDL particles and reduced levels of large HDL particles.

Not only abnormalities of HDL cholesterol discussed in scientific papers concern incorrect subclass distribution but also impaired HDL function. Kalogerakis et al. [23] showed that in type 1 diabetic patients HDL antioxidant function is decreased. In another study [22], authors measured HDL ability to accept an excess of cholesterol from cells that is cholesterol efflux. It has been shown that, despite of abnormalities in HDL subclass distribution and poor metabolic control in patients with DM1, HDL function assessed by cholesterol efflux was comparable to healthy persons. Knowledge of HDL subclasses and its function may help comprehend the mechanisms responsible for vascular disease and chronic diabetes complications and aid in implementing interventions.

\section{Summary}

Knowledge on HDL cholesterol has been extended, however, there are still unknown facts, especially concerning HDL, DM1 and insulin therapy.

It is known that HDL is responsible for the transport of cholesterol to the liver. It is called the "good cholesterol" due to its advantageous properties. There are several subtypes of $\mathrm{HDL}$, depending on the content of apolipoprotein A-I and A-II. We do not know what is their role in patients with DM1. HDL cholesterol reduces cardiovascular risk by its anti-inflammatory, antioxidant, profibrinolytic and antithrombotic effects. The influence of HDL on the incidence of chronic complications of DM1 has not been yet fully understood.

There are known factors that increase the concentration of HDL lipoproteins. These are: physical activity, female sex, estrogens, and glucocorticoids. Factors responsible for the decrease in its concentration 
are: obesity, smoking cigarettes or male gender. Are there other factors affecting the increase or decrease of lipoprotein HDL and its composition in patients with DM1?

The size of HDL affect several enzymes and proteins such as: LCAT, CETP, HL and PLA2. We do not know how in DM1, insulin affects the enzymes involved in the metabolism of HDL, and thus the size of HDL.

The researchers observed an increase in HDL cholesterol level after initiation of insulin therapy. We do not know whether insulin has a direct impact on qualitative and quantitative changes of lipoproteins. It is also unknown, whether insulin promotes the increase of HDL cholesterol only at the start of insulin therapy, or whether this effect is maintained all the time. Moreover, it is not known whether daily insulin requirement and the absolute dose of insulin for the treatment of type DM1 is important. Significant is the fact that insulin affects lipoprotein metabolism in DM1, as it is the treatment of choice of this type of the disease.

\section{REFERENCES}

1. Saboo B. Key elements of successful intensive therapy in patients with type 1 diabetes. Indian J Endocrinol Metab 2015; 19 (suppl 1): S44-S46.

2. Albers JW, Herman WH, Pop-Busui R et al. Effect of prior intensive insulin treatment during the Diabetes Control and Complications Trial (DCCT) on peripheral neuropathy in type 1 diabetes during the Epidemiology of Diabetes Interventions and Complications (EDIC) Study. Diabetes Care 2010; 33: 1090-1096.

3. Zalecenia kliniczne dotyczące postępowania u chorych na cukrzycę 2016. Stanowisko Polskiego Towarzystwa Diabetologicznego. Diabetologia Kliniczna 2016; 5 (suppl A): A8

4. Murray RK, Granner DK, Rodwell VW. Biochemia Harpera. PZWL, Warszawa 2010: 267.

5. Barter PJ, D.o.M. Royal Adelaide Hospital, Adelaide, HDL cholesterol testing: implications for clinical management. Australian Prescriber 1994; 17: 99-102.

6. Kuliszkiewicz-Janus M, Mohamed AS, Abod N. The biology of HDL lipoprotein and its antisclerotic activity. Post Hig Med Dosw (Online) 2006; 60: 307-315.

7. Rosenson RS, Brewer HB, Chapman MJ et al. HDL measures, particle heterogeneity, proposed nomenclature, and relation to atherosclerotic cardiovascular events. Clin Chem 2011; 57: 392-410.

8. Schmitt JK, Harriman K, Poole JR. Modification of therapy from insulin to chlorpropamide decreases HDL cholesterol in patients with non-insulin-dependent diabetes mellitus. Diabetes Care 1987; 10: 692-696.

9. Sinha A, Formica C, Tsalamandris C et al. Effects of insulin on body composition in patients with insulin-dependent and non-insulin-dependent diabetes. Diabet Med 1996; 13: 40-46.

10. Wilson DP, Fesmire JD, Endres RK, Blackett PR. Increased levels of HDL-cholesterol and apolipoprotein A-I after intensified insulin therapy for diabetes. South Med J 1985; 78: 636-638.

11. Guy J, Ogden L, Wadwa RP et al. Lipid and lipoprotein profiles in youth with and without type 1 diabetes: the SEARCH for Diabetes in Youth case-control study. Diabetes Care 2009; 32: 416-420.

12. Araszkiewicz A, Zozulinska-Ziolkiewicz D, Trepinska M, Wierusz-Wysocka B. Knowledge after five-day teaching program in intensive insulin therapy performed at the onset of type 1 diabetes influence the development of late diabetic complications. Diabetes Res Clin Pract 2008; 81: 61-67.

13. Chillaron JJ, Flores-Le-Roux JA, Goday A et al. Metabolic syndrome and type-1 diabetes mellitus: prevalence and associated factors. Rev Esp Cardiol 2010; 63: 423-429.

14. Rogowicz-Frontczak A, Araszkiewicz A, Pilacinski S et al. Carotid intima-media thickness and arterial stiffness in type 1 diabetic patients with and without microangiopathy. Arch Med Sci 2012; 8: 484-490.

15. Witte DR, Tesfaye S, Chaturvedi N et al., Risk factors for cardiac autonomic neuropathy in type 1 diabetes mellitus. Diabetologia 2005; 48: 164-171.

16. Sun JK, Keenan HA, Cavallerano JD et al. Protection from retinopathy and other complications in patients with type 1 diabetes of extreme duration: the joslin 50-year medalist study. Diabetes Care 2011; 34: 968-974.

17. Soedamah-Muthu SS, Vergouwe Y, Costacou T et al. Predicting major outcomes in type 1 diabetes: a model development and validation study. Diabetologia 2014; 57: 2304-2314.

18. Molitch ME, Rupp D, Carnethon M. Higher levels of HDL cholesterol are associated with a decreased likelihood of albuminuria in patients with long-standing type 1 diabetes. Diabetes Care 2006; 29: 78-82.

19. Wierusz-Wysocka B, Zozulinska DA, Araszkiewicz A et al. Higher levels of HDL cholesterol are associated with a decreased likelihood of albuminuria in patients with long-standing type 1 diabetes: Response to Molitch et al. Diabetes Care 2006; 29: 1176-1177.

20. Lyons TJ, Jenkins AJ, Zheng D et al. Diabetic retinopathy and serum lipoprotein subclasses in the DCCT/EDIC cohort. Invest Ophthalmol Vis Sci 2004; 45: 910-918.

21. Tolonen N, Hietala K, Forsblom C et al. Associations and interactions between lipid profiles, retinopathy and nephropathy in patients with type 1 diabetes: the FinnDiane Study. Journal of Internal Medicine 2013; 274: 469-479.

22. Medina-Bravo P, Medina-Urrutia A, Juarez-Rojas JG et al. Glycemic control and high-density lipoprotein characteristics in adolescents with type 1 diabetes. Pediatr Diabetes 2013; 14: 399-406.

23. Kalogerakis G, Baker AM, Christov $S$ et al. Oxidative stress and high-density lipoprotein function in Type I diabetes and end-stage renal disease. Clin Sci (Lond) 2005; 108: 497-506. 